\title{
A Comprehensive Evaluation on Coupling Interaction Characteristics of Generalized Load
}

\author{
Han Shu ${ }^{1, *}$, Dezhi $\mathrm{Li}^{2}$ and Yongxiu $\mathrm{He}^{1}$ \\ ${ }^{1}$ School of Economics and Management, North China Electric Power University, Zhu Xin Zhuang, Bei Nong Road No.2, \\ Changping District, Beijing, China, 102206 \\ ${ }^{2}$ China Electric Power Research Institute, Qinghe, Xiao ying East Road No.15, Haidian District, Beijing, China, 100192 \\ *Corresponding author
}

\begin{abstract}
In order to characterize the interaction between the generalized load and the distribution network in the future, this paper summarizes the interactive mode between the generalized load and the distribution network under the environment of smart power supply first. Then, the evaluation index system and the quantitative analysis method of each index are constructed based on the possible interaction pattern in the future. Finally, this paper puts forward the comprehensive value to describe the interaction between the generalized load and the distribution network by using the TOPSIS method so that we can predict the development trend of the interaction between the generalized load and distribution network.
\end{abstract}

Keywords-component; generalized load; interactive model; interactive feature; comprehensive evaluation

\section{INTRODUCTION}

With the future development of smart power distribution technology, the receiving end will have a wide range of load which can generate power. It mainly includes the load with distributed power supply, the electric vehicle based on V2G technology and the load with energy storage device. In this paper, this kind of load is called "generalized load" [1-3]. The emergence of such a load will break the one-way flow situation that the electricity flows from the distribution network to the user side. There will be a double interaction between the grid and the load in the future. The first interaction is the demand side response, the other one is the real power exchange [4-5]. Aiming at these characteristics, interaction models of the "Grid - Load" are summarized. Then, this paper presents an index system and a quantitative method for describing the characteristics of generalized load interaction. Finally, the TOPSIS method is used to evaluate the index system comprehensively, and the quantitative analysis value of the interaction between the network and the load is obtained.

\section{COUPLING CHARACTERISTICS OF GENERALIZED LOAD}

In the future, with the wide access to the receiving end of the medium and small power system, the traditional load characteristics of the user side will be changed greatly. The interaction between the load and the distribution network will be more frequent, and the complexity of the interaction is manifested in the uncertainty of interaction time and displacement of the interactive space. Not only that, the generalized load can be used as a power supply, there will gradually emerge the characteristics of power coupling among loads. It is this kind of load characteristic of "horizontal and vertical interaction" that makes power consuming behavior more complex, and increases the difficulty of load forecasting work. In this paper, the typical generalized loads are classified as follows.

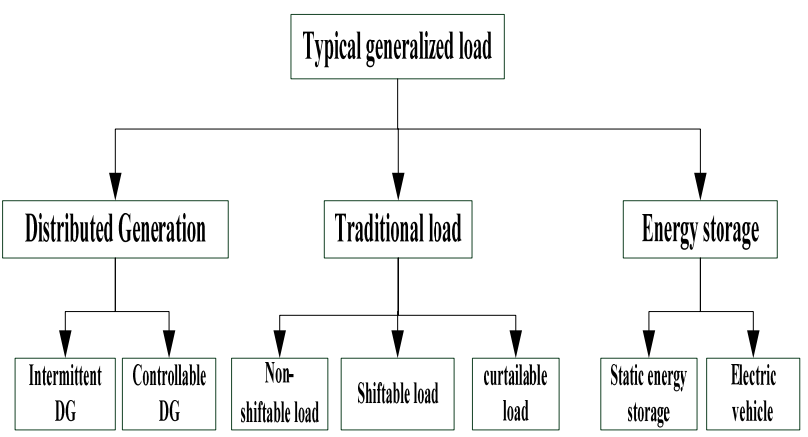

FIGURE I. THE CLASSIFICATION Of TYPICAL GENERALIZED LOAD

In the future, the interaction between the generalized load and the power grid can be based on the interaction of the excitation signal and the substantial power exchange. It can be seen that the shift able load and curtail able load can adjust their power consumption behavior according to excitation signals of demand response. The interaction of substantial power exchange refers to the bidirectional exchange between distribution network and generalized load according to its own energy characteristics. In addition, generalized load itself based on the power attributes can achieve the coupling complementary of power in time and space. Distributed power supply can be used for users first; the surplus can be stored in an energy storage device or sold through a large grid. At the peak of power consumption, the power supply of energy storage devices can be released when the distributed power supply is insufficient so that it can realize the coupling among loads. 


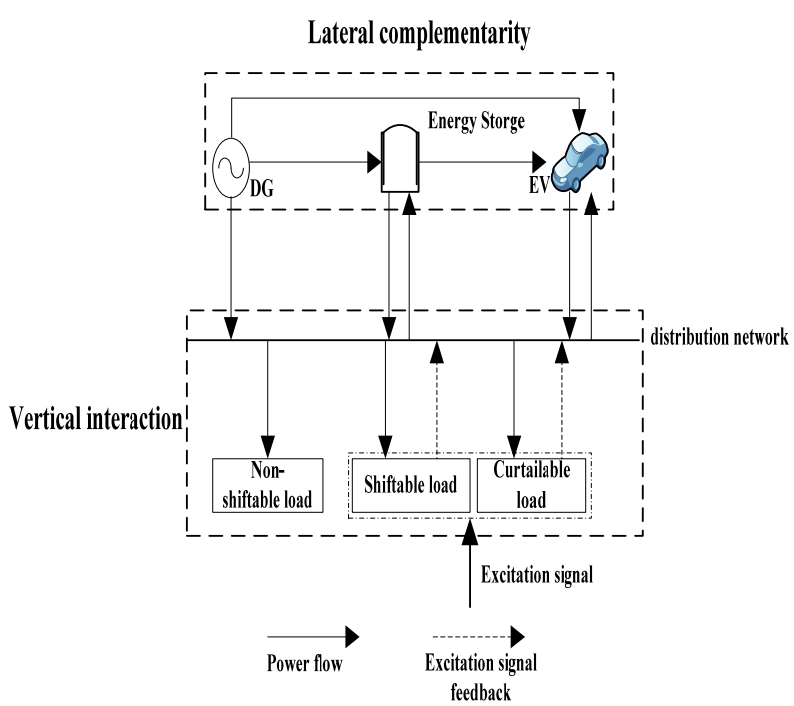

FIGURE II. GENERALIZED LOAD COUPLING INTERACTION MODEL

\section{INTERACTION INDEX SYSTEM CONSTRUCTION OF GENERALIZED LOAD}

In order to depict the interaction characteristics of the generalized load, this paper constructs the index system of interactive features, and puts forward the specific quantitative methods of each index. It can measure the interaction ability of generalized load through the specific level value of each index. The interactive index system is shown in the following table 1:

\section{A. Supply Rate of Load with Power Attribute during Peak Period}

In order to measure the future development trend of generalized load, this paper constructs the index, which is called supply rate of load with power attribute at peak period. This indicator is expressed by the ratio of the total power output of load with power attribute and total load demand during the peak period. The greater the value is, the greater the proportion of the load with the power attribute will be. Where $R_{\mathrm{SE}}$ stands supply rate of load with power attribute at peak period; $P(t)$ expresses total power output of load with power attribute during peak period; $L(t)$ indicates total load demand during peak period. $T_{\text {peak }}$ represents peak hours.

$$
R_{\mathrm{SE}}=\frac{\int_{t \in \mathrm{T}_{\text {peak }}} P(t) d t}{\int_{\mathrm{t} \in \mathrm{T}_{\text {peak }}} L(t) d t} \times 100 \%
$$

TABLE I. GENERALIZED LOAD INTERACTION INDEX SYSTEM

\begin{tabular}{|c|c|c|c|}
\hline \multicolumn{1}{|c|}{ Item } & Index & Calculation formula & Note \\
\hline \multirow{2}{*}{$\begin{array}{c}\text { Generalized load } \\
\text { interaction index }\end{array}$} & Supply rate of load with power attribute at peak period & Formula (1) & Maximum Type Index \\
\cline { 2 - 4 } & Reverse power supply capability of load with power attribute & Formula (2) & Maximum Type Index \\
\cline { 2 - 4 } & Generalized load interaction rate & Formula (3) & Maximum Type Index \\
\cline { 2 - 4 } & Lateral complementarities capability of generalized load & Formula (4) & Maximum Type Index \\
\hline
\end{tabular}

\section{B. Reverse Power Supply Capability of Load with Power} Attribute

In this paper, reverse power supply capability of load with power attribute is used to measure the interaction intensity between the generalized load and distribution network. The index is expressed by the ratio of the electricity delivered to distribution network by load with power attribute and total power consumption. The larger the value is, the stronger the interaction strength of generalized load will be. Where ROP is reverse power supply capability of load with power attribute; $P_{\mathrm{OP}}(t)$ indicates reverse output of load with power supply attributes.

$$
R_{\mathrm{OP}}=\frac{\int_{0}^{23} P_{\mathrm{OP}}(t) d t}{\int_{0}^{23} L(t) d t}
$$

\section{Generalized Load Interaction Rate}

In this paper, the interaction rate between the generalized load and the distribution network is measured by generalized load interaction rate. The index can be expressed by the ratio of the total reverse power supply by load with power attribute and the duration of peak period. The larger the value is, the faster 
the speed of generalized load interaction will be. Where $V_{\text {peak }}$ indicates generalized load interaction response rate.

$$
V_{\text {peak }}=\frac{\int_{\mathrm{t} \in \mathrm{T}_{\text {peak }}} P_{\mathrm{OP}}(t) d t}{T_{\text {peak }}} \times 100 \%
$$

\section{Lateral Complementarities Capability of Generalized Load}

The paper describes the degree of synergy among generalized load by means of the lateral complementary capacity of generalized load. The index is expressed by the proportion of the distributed power supply absorbed by the energy storage device to the total distributed output. The larger the value is, the stronger the lateral complementary ability of the generalized load is. Where $C_{\mathrm{DR}}$ is Lateral complementarities capability of generalized load; $P_{\text {storage }}(t)$ expresses the power of energy storage equipments when absorbing distributed power output; $P_{\mathrm{DRE}}(\mathrm{t})$ stands for generation power of distributed generation.

$$
\mathrm{C}_{\mathrm{D} \mathrm{R}}=\frac{\int_{0}^{23} P_{\text {storge }}(t) d t}{\int_{0}^{23} P_{\mathrm{D} \mathrm{RE}}(t) d t}
$$

\section{COMPREHENSIVE EVALUATION MODEL OF GENERALIZED LOAD INTERACTION BASED ON TOPSIS METHOD}

According to the above index system, in order to comprehensively measure the generalized load coupling characteristics, this paper uses the Technique for Order Preference by Similarity to an Ideal Solution (TOPSIS) to evaluate the above indexes. When using TOPSIS method, it needs to set up an ideal system or sample point such as $\left(x^{*}{ }_{i 1}\right.$, $\left.x^{*}{ }_{\mathrm{i} 2} \ldots x^{*}{ }_{\mathrm{im}}\right)$. We can define the weighted distance between it and the evaluated object $\left(\mathrm{x}_{\mathrm{i} 1}, \mathrm{x}_{\mathrm{i} 2} \ldots \mathrm{x}_{\mathrm{im}}\right)$ as follows:

$$
y_{i}=\sum_{j=1}^{m} w_{j} f\left(x_{\mathrm{ij}}, x_{j}^{*}\right), i=1,2, \ldots, n
$$

where $w_{j}$ indicates weight coefficient; $f\left(x_{\mathrm{ij}}, x_{\mathrm{j}}^{*}\right)$ expresses a kind of distance, Euclidean distance is usually used as a composite function. Setting $\left(x^{+}{ }_{1}, X^{+}{ }_{2} \ldots X^{+}{ }_{m}\right)$ is a positive ideal system and $\left(x_{1}^{-}, x_{2}^{-} \ldots x_{\mathrm{m}}^{-}\right)$is a negative ideal system. According to the weighted distance formula, we can calculate the Euclidean distance of each scheme from the positive and negative ideal system. After calculating the Euclidean distance, the formula (4) can be used to calculate the queuing indication value. The greater the value is, the better the results will be. Specific formulas are as follows:

Distance formula to positive ideal point:

$$
y_{i}^{+}=\sum_{j=1}^{m} w_{j}\left(x_{i j}-x_{j}^{+}\right)^{2}(i=1,2,3 \ldots, n)
$$

Distance formula to negative ideal point:

$$
y_{i}^{-}=\sum_{j=1}^{m} w_{j}\left(x_{i j}-x_{j}^{-}\right)^{2}(i=1,2,3 \ldots, n)
$$

Queuing indication value formula:

$$
C_{i}=\frac{y_{i}^{-}}{y_{i}^{+}+y_{i}^{-}}
$$

Using the above evaluation model, the positive and negative ideal value of each quantitative index is set up. Combined with the generalized load correlation data, we can get a queuing indicator value to describe the coupling interaction characteristics of generalized load. If the value is big enough, we can believe that the ability of coupling interaction is very strong. At the same time, the value of the index is not the same in different stages of development, so it can be used to describe the development of generalized load in smart electricity environment.

\section{CONCLUSION}

This paper first summarizes the interactive coupling characteristics of generalized load in the future smart electrical environment. Secondly, to describe the interactive characteristics of generalized load, an index system is proposed through combining with the specific interaction modes of generalized load. Finally, according to the above index system, the TOPSIS method is put forward to evaluate the comprehensive interaction characteristics of the generalized load, which could be measured by using the queuing indication value. Based on the above analysis, conclusions of this paper are as follows:

With the development of intelligent transmission and distribution technology, there will be a wide range of generalized load accessing into the grid in the future. From a vertical perspective, the generalized load will produce many types of flexible interactions with the grid, including responsebased demand-side management interactions and essential energy exchange that regulates its own energy level. From a horizontal perspective, the coupling complementary between the loads with power supply attribute will appear, and so as the complicated behaviour of energy using modes.

The generalized load itself owns power supply properties, such load will show dual role in the future, that is, involving in the demand side response and adjusting their habits flexibly. Thus, it is necessary to establish a comprehensive evaluation index system to describe the coupling interaction characteristics of generalized load. 


\section{REFERENCES}

[1] Guoxia Sun, Jian Zhang, Haijiang Wu, "Research on the identification and adaptation of generalized load modelling based on distributed generators," Power System Protection and Control, vol. 41, pp. 105-111, 2013.

[2] Xionghua Shi, Genjun Chen, Ping Ju, "A new generalized power system load model and its application," JOURNAL OF ELECTRIC POWER SCIENCE AND TECHNOLOGY, vol. 26, pp.44-48, October 2011.

[3] Qingfeng Tang, Nian Liu, Jianhua Zhang, "Theory and key problems for automated demand response of user side considering generalized demand side resources," Power System Protection and Control, vol. 42, pp. 138-147, December 2014.

[4] Jianguo Yao, Shengchun Yang, Ke Wang, "Concept and Research Framework of Smart Grid 'Source-Grid-Load' Interactive Operation and Control," Automation of Electric Power systems, vol. 36, pp. 1-6, 12, June 2012.

[5] Shengchun Yang, Jiantao Liu, Jianguo Yao, "Model and Strategy for Multi-time Scale Coordinated Flexible Load Interactive Scheduling," Proceedings of the CSEE, vol.34, pp. 3664-3673 , August 2014.

[6] Long Xing, Peichao Zhang, Chen Fang, "Optimal Operation for Microgrid Using Generalized Demand Side Resources," Automation of Electric Power system, vol. 37, pp. 7-12, 133, December 2013.

[7] Yanbin Li, Xinyi Yu, Zhijie Wang, "Risk Assessment on Photovoltaic Power Generation Project by Grey Correlation Analysis and TOPSIS Method,” Power System Technology, vol. 37, pp.1514-1519, June 2013. 